\title{
New resistorless tunable voltage-mode universal filter using single VDIBA
}

\author{
NORBERT HERENCSAR, OGUZHAN CICEKOGLU, ROMAN SOTNER, \\ JAROSLAV KOTON, KAMIL VRBA
}

Citation: Analog Integrated Circuits and Signal Processing, vol. 76, no. 2, pp. 251-260

ISSN: 0925-1030 (Print)

ISSN: 1573-1979 (Online)

DOl: http://dx.doi.org/10.1007/s10470-013-0090-2

Accepted manuscript

The final publication is available at Springer via http://dx.doi.org/10.1007/s10470-013-0090-2 


\title{
New resistorless tunable voltage-mode universal filter using single VDIBA
}

\author{
Norbert Herencsar • Oguzhan Cicekoglu • \\ Roman Sotner • Jaroslav Koton • Kamil Vrba
}

Received: date / Accepted: date

\begin{abstract}
To increase the universality of the recently introduced voltage differencing inverting buffered amplifier (VDIBA), this letter presents a new voltage-mode (VM) multi-input-single-output (MISO) universal filter. The proposed filter contains only single VDIBA, two capacitors, and one nMOS transistor, operated in triode region, and is used for resonance angular frequency tuning. Since in the structure no resistors are needed the filter can be classified as resistorless. The VM MISO filter compared with other active building block-based counterparts is very simple, it contains only few transistors, and has the smallest size area. Moreover, no component matching is required and it shows low sensitivity performance. The theoretical results are verified by SPICE simulations using TSMC $0.18 \mu \mathrm{m}$ level-7 SCN018 CMOS process parameters with $\pm 0.9 \mathrm{~V}$ supply voltages. In addition, the behavior of the proposed VM filter was also experimentally verified using commercially available integrated circuits OPA660 and AD830.
\end{abstract}

N. Herencsar $(\bowtie) \cdot$ J. Koton $\cdot$ K. Vrba

Department of Telecommunications, Brno University of Technology, Technicka 3082/12, 61600 Brno, Czech Republic

E-mail: herencsn@feec.vutbr.cz

J. Koton

E-mail: koton@feec.vutbr.cz

K. Vrba

E-mail:vrbak@feec.vutbr.cz

O. Cicekoglu

Department of Electrical and Electronics Engineering, Bogazici University, 34342-Bebek, Istanbul, Turkey

E-mail: cicekogl@boun.edu.tr

R. Sotner

Department of Radio Electronics, Brno University of Technology, Technicka 3082/12, 61600 Brno, Czech Republic

E-mail: sotner@feec.vutbr.cz
Keywords Active filter · Multi-input single-output (MISO) · Universal filter · Resistorless filter · Voltagemode $\cdot$ Voltage differencing inverting buffered amplifier (VDIBA)

\section{Introduction}

Active filters are widely used as basic building blocks in analog signal processing. They are intended to replace the RLC filters that mainly suffer from the lack of integrability. Such active filter circuits are successfully used in video signal processing, communication systems, computer systems, telephone circuitry, broadcasting systems, control and instrumentation systems, etc. [1]. Recently, several active building block (ABB)based minimal configuration voltage-mode (VM) multiinput single-output (MISO) universal filter structures have been reported in the literature in which for specific connection both capacitors are used as input terminal [2]-[13]. Unfortunately, the internal structures of ABBs in all of above reported filters suffer from the excessive number of transistors and the complexity of the used ABBs, which results in high silicon area in case of onchip fabrication. This can be also seen from Table 1, which summarizes the advantages and disadvantages of previously reported VM MISO filters in [2]-[13] based on various relevant criteria. Full nomenclature of referred ABBs is given in Appendix. With the aim to minimize the silicon area on the chip, very recently the new versatile $\mathrm{ABB}$ so-called voltage differencing inverting buffered amplifier (VDIBA) has been presented [14]. Its application possibilities have been shown on the design of a resistorless and electronically tunable dual-output VM first-order all-pass filter [14], four-phase quadrature oscillator, second-order all-pass filter [15], single ended 
Table 1 Comparison of performance specifications of different VM MISO filters

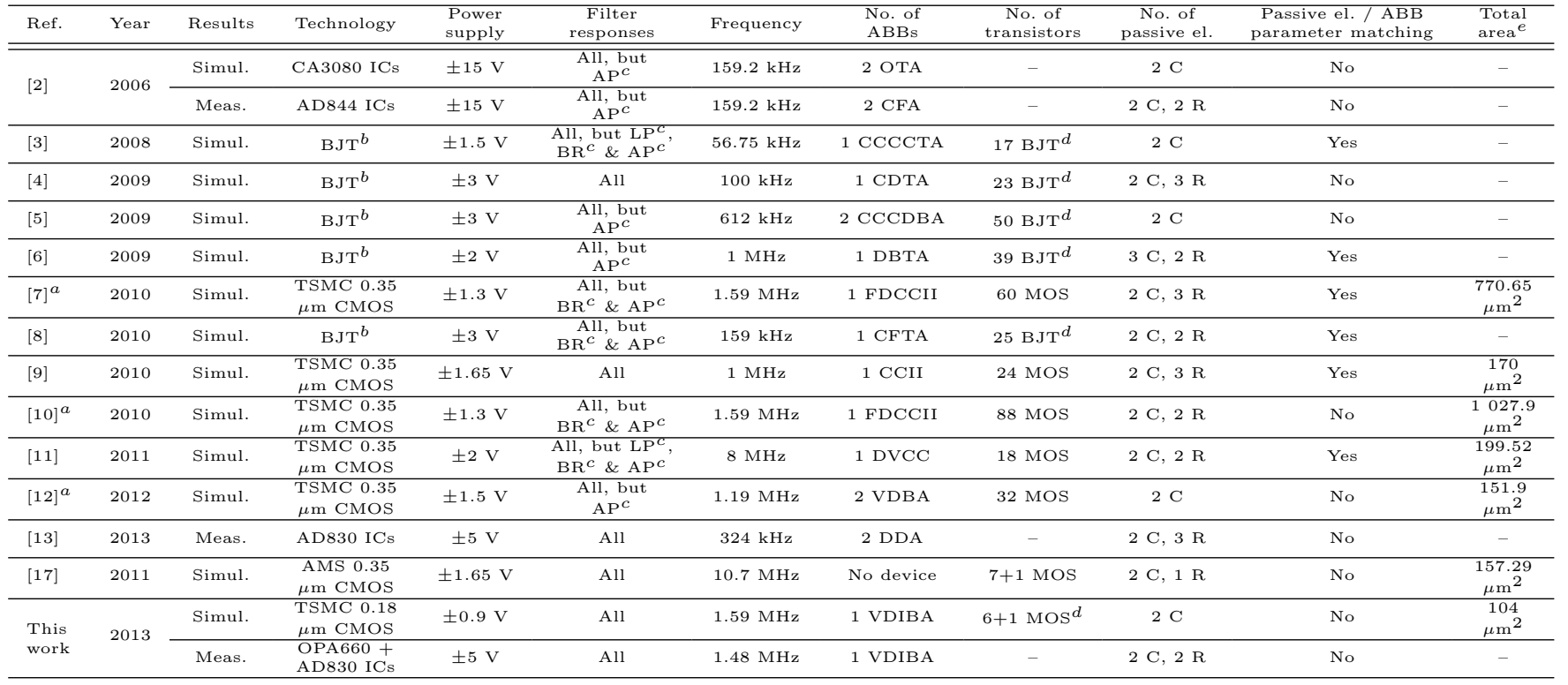

Notes:

$\bar{a}$ Not applicable

[10] Fig. 4; [12] Fig. 3

(N parameters NR100N or NR200N (NPN) and PR100N or PR200N (PNP) of bipolar arrays ALA400 from AT\&T

$c$ For realization additional inverting voltage input needed

d Ideal current source(s) assumed

Sum of products of the lengths and widths of each transistors in used CMOS structure incl. VCR area

and differential mode triangle/square wave generators [16].

In this letter we propose a new VM MISO universal filter using the just mentioned VDIBA as active element. The proposed filter structure is an alternative realization to recently published CMOS-RC VM MISO universal filter [17] and compared with other ABB-based counterparts in the literature [2]-[13] and CMOS-RC [17], it shows the following advantages:

(i) compared to circuits in [2], [3], [5]-[8], and [10]-[12] it realizes all five standard filter functions i.e. lowpass filter (LP), band-pass (BP), high-pass (HP), band-reject (BR), and all-pass (AP) without the need of additional inverting voltage inputs, which lead to higher complexity of these solutions,

(ii) proposed filter is resistorless i.e. no external resistor is needed, a useful feature that cannot be found in $[2],[4],[6]-[11],[13]$, and [17],

(iii) it employs only few transistors in total, hence the total transistor area is much smaller than [2]-[13] and $[17]$

(iv) no passive element or ABB parameter matching is required in the design, which is not available in [3], [6]-[9], and [11],

(v) suitable for low-voltage operation, while circuits [2][13] and [17] are supplied with $\pm 1.3 \mathrm{~V}$ or higher DC voltages.
SPICE simulation results based on Taiwan Semiconductor Manufacturing Company (TSMC) $0.18 \mu \mathrm{m}$ level-7 SCN018 CMOS process parameters and experimental measurement results using readily available integrated circuits (ICs) OPA660 and AD830 are included to support the theory.

\section{Circuit description}

The VDIBA is a recently introduced four-terminal active device with circuit symbol shown in Fig. 1 [15]. It has a pair of high-impedance voltage inputs $v+$ and $v-$, a high-impedance current output $z$, and low-impedance voltage output $w-$. The input stage of VDIBA can be easily implemented by a differential-input single-output operational transconductance amplifier (OTA), which converts the input voltage to output current that flows out of the $z$ terminal. The output stage can be formed by unity-gain inverting voltage buffer (IVB). Since both stages can be implemented by commercially available

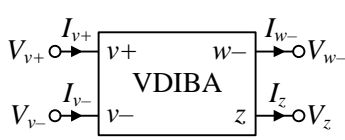

Fig. 1 Circuit symbol of VDIBA. 


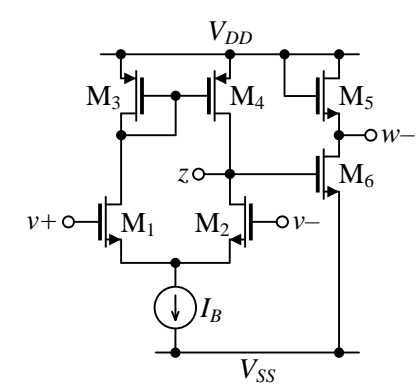

Fig. 2 CMOS implementation of VDIBA.

ICs, and moreover it contains OTA whose $g_{m}$ can be electronically controllable via DC bias current, the introduced active element is attractive for resistorless and electronically controllable circuit applications.

Using standard notation, the relationship between port currents and voltages of the VDIBA can be described by following hybrid matrix:

$$
\left[\begin{array}{c}
I_{v+} \\
I_{v-} \\
I_{z} \\
V_{w-}
\end{array}\right]=\left[\begin{array}{cccc}
0 & 0 & 0 & 0 \\
0 & 0 & 0 & 0 \\
g_{m} & -g_{m} & 0 & 0 \\
0 & 0 & -1 & 0
\end{array}\right]\left[\begin{array}{c}
V_{v+} \\
V_{v-} \\
V_{z} \\
I_{w-}
\end{array}\right],
$$

where $g_{m}$ represents the transconductance of VDIBA.

The CMOS implementation of the VDIBA is shown in Fig. 2. The circuit is composed of an active loaded differential pair (transistors $\mathrm{M}_{1}-\mathrm{M}_{4}$ ) followed by a unitygain IVB (matched transistors $\mathrm{M}_{5}$ and $\mathrm{M}_{6}$ ). The input/output terminal resistances of the CMOS VDIBA shown in Fig. 2 can be found as:

$R_{o w-} \cong \frac{1}{g_{m 5}} \| r_{o 6}$

$R_{o z} \cong r_{o 4} \| r_{o 2}$

$R_{v+}=R_{v-} \cong \infty$

where $g_{m i}$ and $r_{o i}$ represents the transconductance and output resistance of the $i$-th transistor, respectively. From Eqs. (2)-(4) it can be seen that the output terminal $(w-)$ can exhibit low resistance by selecting large transistor $\mathrm{M}_{5}$ (and $\mathrm{M}_{6}$ due to the matching condition requirement), the input terminals ( $v+$ and $v-$ ), as well as the $z$ terminal, have high resistances.

The proposed VM MISO universal filter is shown in Fig. 3. The circuit employs single VDIBA as active element, two capacitors, and one nMOS transistor working as voltage-controlled resistor (VCR). Although in practice, filters employing only grounded capacitors are preferred, new IC technologies offer floating capacitor realization possibility as a double poly (poly1poly2) or metal-insulator-metal (MIM) capacitor [18].

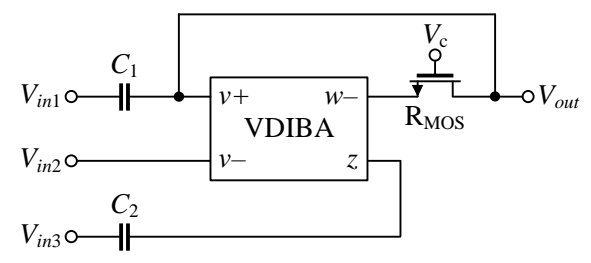

Fig. 3 Proposed VM universal filter.

The nMOS transistor works in triode region and its resistance $R_{\mathrm{MOS}}$ for low value of signal amplitudes can be calculated as follows:

$R_{\mathrm{MOS}} \cong \frac{1}{\mu_{\mathrm{n}} C_{\mathrm{ox}} \frac{W}{L}\left(V_{\mathrm{c}}-V_{\mathrm{THn}}\right)}$,

where $C_{\mathrm{ox}}$ is the gate oxide capacitance per unit area, $\mu_{\mathrm{n}}$ is the free electron mobility in the channel, $W$ and $L$ are the channel width and length of the nMOS, $V_{\mathrm{THn}}$ is the threshold voltage of the transistor, and $V_{\mathrm{c}}$ is DC control voltage used for tuning. Using (1), circuit analysis yields the following output voltage $V_{\text {out }}$ of the proposed circuit in Fig. 3:

$V_{\text {out }}=\frac{s^{2} C_{1} C_{2} R_{\mathrm{MOS}} V_{i n 1}-s C_{2} V_{i n 3}+g_{m} V_{i n 2}}{s^{2} C_{1} C_{2} R_{\mathrm{MOS}}+s C_{2}+g_{m}}$.

From Eq. (6) it can be observed that the proper connection of the relevant input terminals yields the five standard types of biquadratic filter functions as follows:

(i) If $V_{i n 1}=V_{i n 3}=0$ (grounded), a second-order LP filter can be obtained with $V_{\text {out }} / V_{\text {in } 2}$;

(ii) If $V_{i n 1}=V_{i n 2}=0$ (grounded), a second-order inverting BP filter can be obtained with $V_{\text {out }} / V_{\text {in } 3}$;

(iii) If $V_{i n 2}=V_{i n 3}=0$ (grounded), a second-order HP filter can be obtained with $V_{\text {out }} / V_{\text {in } 1}$;

(iv) If $V_{i n 3}=0$ (grounded) and $V_{i n 1}=V_{i n 2}=V_{i n}$, a second-order BR filter can be obtained with $V_{\text {out }} / V_{\text {in }}$;

(v) If $V_{i n 1}=V_{i n 2}=V_{i n 3}=V_{i n}$, a second-order AP filter can be obtained with $V_{\text {out }} / V_{\text {in }}$.

Thus, the circuit is capable of realizing LP, BP, HP, $\mathrm{BR}$, and AP responses from the same topology without any requirement for component-matching conditions or use of additional inverting voltage inputs. For all filter responses the resonance angular frequency $\omega_{0}$, quality factor $Q$, and bandwidth $\omega_{0} / Q$ derived from the denominator of Eq. (6) are:

$$
\begin{aligned}
& \omega_{0}=\sqrt{\frac{g_{m}}{C_{1} C_{2} R_{\mathrm{MOS}}}}, \\
& Q=\sqrt{\frac{C_{1} g_{m} R_{\mathrm{MOS}}}{C_{2}}}, \\
& \frac{\omega_{0}}{Q}=\frac{1}{C_{1} R_{\mathrm{MOS}}} .
\end{aligned}
$$


It should be noted that the gain factors for all five filter responses are equal to unity in magnitude.

A sensitivity study forms an important index of the performance of any active network. The active and passive relative sensitivities of the proposed circuit derived from (7)-(9) are given as:

$S_{g_{m}}^{\omega_{0}}=-S_{C_{1}, C_{2}, R_{\mathrm{MOS}}}^{\omega_{0}}=\frac{1}{2}, \quad S_{C_{1}, g_{m}, R_{\mathrm{MOS}}}^{Q}=-S_{C_{2}}^{Q}=\frac{1}{2}$,

$S_{C_{1}, R_{\mathrm{MOS}}}^{\omega_{0} / Q}=-1$.

From the results it is evident that the sensitivities are low and not larger than unity in absolute value.

\section{Stability analysis}

Considering the non-idealities of the VDIBA, the matrix relationship of Eq. (1) converts to:

$$
\left[\begin{array}{c}
I_{v+} \\
I_{v-} \\
I_{z} \\
V_{w-}
\end{array}\right]=\left[\begin{array}{cccc}
0 & 0 & 0 & 0 \\
0 & 0 & 0 & 0 \\
g_{m}(s) & -g_{m}(s) & s C_{z}+1 / R_{z} & 0 \\
0 & 0 & -\beta(s) & R_{w-}
\end{array}\right]\left[\begin{array}{c}
V_{v+} \\
V_{v-} \\
V_{z} \\
I_{w-}
\end{array}\right],
$$

where the parasitic impedance $R_{z}$ is ideally infinity as well as $C_{z}$ and $R_{w}$ - are ideally equal to zero and the $\beta$ and $g_{m}$ are non-ideal voltage and transconductance error gains of VDIBA, respectively.

Considering the effects of these non-ideal gains on the proposed filter, the output voltage $V_{\text {out }}$ in Eq. (6) turns to:

$V_{\text {out }} \cong \frac{s^{2} C_{1} C_{2} R_{\mathrm{MOS}} V_{\text {in } 1}-\beta s C_{2} V_{\text {in } 3}+\beta g_{m} V_{\text {in } 2}}{s^{2} C_{1} C_{2} R_{\mathrm{MOS}}+s C_{2}+\beta g_{m}}$.

It should be mentioned that non-ideal gains $\beta$ and $g_{m}$ are frequency-dependent parameters that using a single-pole model [19] can be defined as follows:

$\beta(s)=\frac{\beta_{o}}{1+s \tau_{\beta}}$,

$g_{m}(s)=\frac{g_{m o}}{1+s \tau_{g_{m}}}$,

where $\beta_{o}=1-\varepsilon_{\beta_{v}}$ and $g_{m o}=g_{o}\left(1-\varepsilon_{g_{m}}\right)$ are DC voltage and transconductance gains of VDIBA, respectively. Ideally, the voltage gain $\beta_{o}$ is equal to unity. Considering the VDIBA implementation using internal structure shown in Fig. 2, at low and medium frequencies these DC gains can be assumed to be constants with following values of tracking errors obtained by SPICE simulations (for $I_{B}=100 \mu \mathrm{A}$ ): $\varepsilon_{\beta_{v}}=0.078$ and $\varepsilon_{g_{m}}=0.004$, respectively, while $g_{o}=600 \mu \mathrm{A} / \mathrm{V}$. In addition, $\tau_{\beta}=1 / \omega_{\beta}$ and $\tau_{g_{m}}=1 / \omega_{g_{m}}$ in which $\omega_{\beta}$ and $\omega_{g_{m}}$ are $-3 \mathrm{~dB}$ cut-off frequency of the corresponding gain responses found to be $f_{\beta-3 \mathrm{~dB}}=51.93$ $\mathrm{GHz}$ and $f_{g_{m}-3 \mathrm{~dB}}=226.32 \mathrm{MHz}$. Hence, the maximum operating frequency of the VDIBA is $f_{\max } \ll(1 / 2 \pi) \times$ $\min \left\{\omega_{\beta}, \omega_{g_{m}}\right\} \approx 226.32 \mathrm{MHz}$. As it can be seen the $\tau_{\beta}$ is much smaller than $\tau_{g_{m}}$. This is due to the fact that the unity-gain IVB stage $\left(\mathrm{M}_{5}\right.$ and $\left.\mathrm{M}_{6}\right)$ can achieve a much higher bandwidth because its output impedance is quite small, ideally equal to zero [20].

The best way to test the stability of the proposed filter characteristics is the use of Routh-Hurwitz stability criterion [21]. Thus, assuming that the single-pole model analysis gives satisfactory result and neglecting the effect of $\tau_{\beta}$, by replacing Eq. (14) into (12) its denominator turns into the following third-order polynomial:

$D(s)=\sum_{i=0}^{3}\left(a_{i} s^{i}\right)$.

Here, the coefficients of $a_{i}(i=0,1,2,3)$ are calculated as:

$a_{0}=\beta_{o} g_{m o}$

$a_{1}=C_{2}$,

$a_{2}=C_{2}\left(C_{1} R_{\mathrm{MOS}}+\tau_{g_{m}}\right)$,

$a_{3}=C_{1} C_{2} R_{\mathrm{MOS}} \tau_{g_{m}}$

Thus, in order to prevent stability problems, the following condition should be satisfied:

$\tau_{g_{m}}<\frac{C_{1} C_{2} R_{\mathrm{MOS}}}{\beta_{o} C_{1} g_{m o} R_{\mathrm{MOS}}-C_{2}}$.

\section{Performance verifications}

\subsection{Simulation results}

To verify the theoretical study, the behavior of the proposed VDIBA-based VM filter shown in Fig. 3 has been verified by SPICE simulations with DC power supply voltages equal to $+V_{D D}=-V_{S S}=0.9 \mathrm{~V}$. In the design, transistors are modeled by the TSMC $0.18 \mu \mathrm{m}$ level- 7 SCN018 CMOS process parameters $\left(V_{\mathrm{THn}}=0.3725 \mathrm{~V}\right.$, $\mu_{\mathrm{n}}=259.5304 \mathrm{~cm}^{2} /(\mathrm{V} \cdot \mathrm{s}), V_{\mathrm{THp}}=-0.3948 \mathrm{~V}, \mu_{\mathrm{p}}=$ $\left.109.9762 \mathrm{~cm}^{2} /(\mathrm{V} \cdot \mathrm{s}), T_{\mathrm{ox}}=4.1 \mathrm{~nm}\right)[22]$. The aspect ratios of the OTA $\left(\mathrm{M}_{1}-\mathrm{M}_{4}\right)$ and the $\operatorname{IVB}\left(\mathrm{M}_{5}\right.$ and $\left.\mathrm{M}_{6}\right)$ were chosen as $W / L_{(\mathrm{M} 1-\mathrm{M} 4)}=18 \mu \mathrm{m} / 1.08 \mu \mathrm{m}$ and $W / L_{(\mathrm{M} 5, \mathrm{M} 6)}=54 \mu \mathrm{m} / 0.18 \mu \mathrm{m}$, respectively. Note that the bulk of all transistors is connected to their corresponding sources to prevent body effect and $W / L$ ratio of the transistors $\mathrm{M}_{5}$ and $\mathrm{M}_{6}$ were selected sufficiently high to decrease the loading effect. 


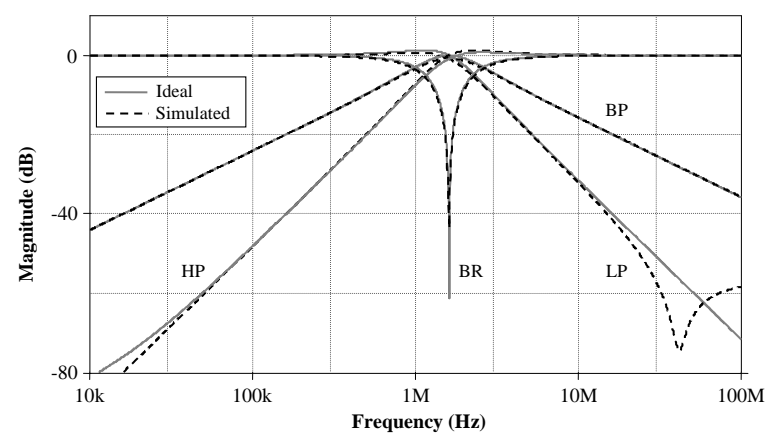

(a)

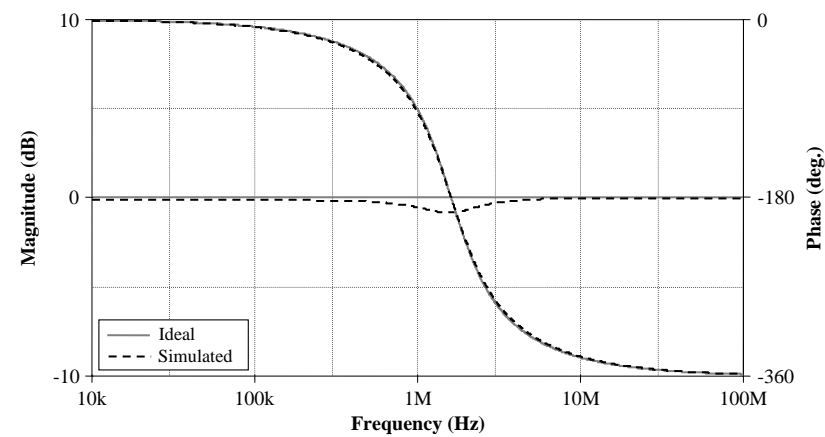

(b)

Fig. 4 (a) Ideal and simulated magnitude responses of the low-pass, band-pass, high-pass, and band-reject filters, (b) ideal and simulated magnitude and phase responses of the all-pass filter in Fig. 3.

The proposed filter was designed for $f_{0}=\omega_{0} / 2 \pi \cong$ $1.59 \mathrm{MHz}$ and $Q=1$ by choosing $C_{1}=C_{2}=60 \mathrm{pF}$, $I_{B}=100 \mu \mathrm{A}\left(g_{m}=600 \mu \mathrm{A} / \mathrm{V}\right)$, and $R_{\mathrm{MOS}}=1.65 \mathrm{k} \Omega$ obtained with aspect ratio $W / L=6.3 \mu \mathrm{m} / 1.08 \mu \mathrm{m}$ and DC control voltage $V_{\mathrm{c}}=0.84 \mathrm{~V}$. Figure $4(\mathrm{a})$ shows the ideal and simulated magnitude responses of the lowpass, band-pass, high-pass, and band-reject filters of Fig. 3. Similarly, the ideal and simulated magnitude and phase responses of the all-pass filter are shown in Fig. 4(b). Thus, from Fig. 4(a) and (b) it can be observed that the proposed circuit is capable of realizing all the five standard filter functions as expected. Also, the time-domain response of the proposed filter in Fig. 3 is investigated by applying a sinusoidal input voltage signal with an amplitude of $50 \mathrm{mV}$ peak at $f_{0}=1.59 \mathrm{MHz}$. Figure 5 shows the input and output signals of the band-pass response of the proposed filter. The total harmonic distortion (THD) of the output signal is found to be $3.15 \%$.

To demonstrate electronic tunability of the proposed VM filter, pole frequency is tuned around the expected frequency of $1.59 \mathrm{MHz}$ by varying DC control voltage $V_{\mathrm{c}}$ of VCR $R_{\mathrm{MOS}}$ for a high- $Q$ band-pass response of the filter. In this case, for $Q=5$ the component

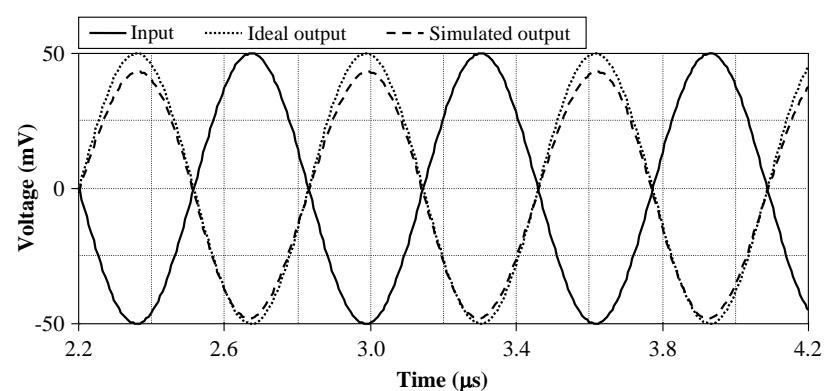

Fig. 5 The output signal of the band-pass response with $Q=$ 1 of the filter in Fig. 3 applying a $50 \mathrm{mV}$ (peak) sinusoidal input signal at $1.59 \mathrm{MHz}$.

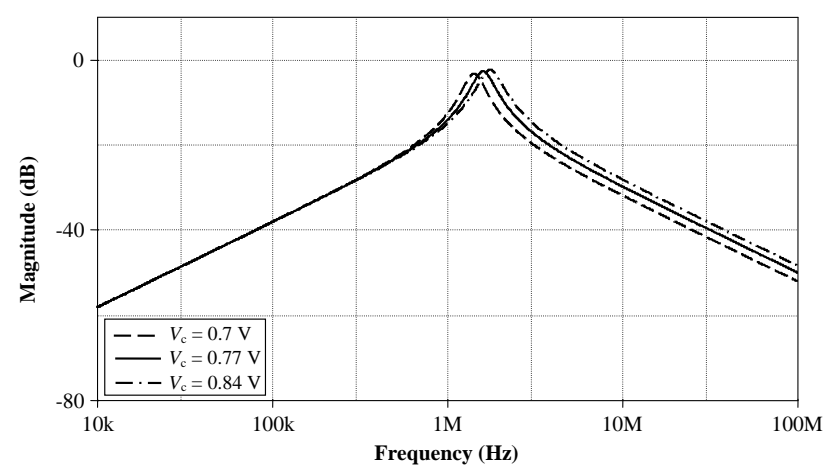

Fig. 6 Electronically tuned high- $Q$ band-pass magnitude responses.

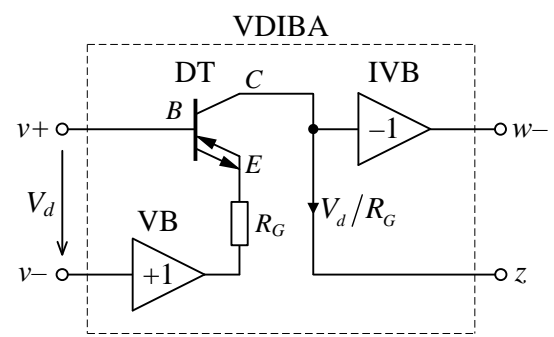

Fig. 7 VDIBA implementation using commercially available ICs.

values were selected as $C_{1}=250 \mathrm{pF}, C_{2}=8 \mathrm{pF}$, $I_{B}=52 \mu \mathrm{A}\left(g_{m}=400 \mu \mathrm{A} / \mathrm{V}\right)$, and $R_{\mathrm{MOS}}=2 \mathrm{k} \Omega$ obtained with above given aspect ratio and DC control voltage $V_{\mathrm{c}}=0.77 \mathrm{~V}$. By slightly varying $V_{\mathrm{c}}$ as it is demonstrated in Fig. 6, the changes in $R_{\mathrm{MOS}}$ will be small. Since the value of the quality factor is proportional to the square root of $R_{\mathrm{MOS}}$, its value will not be seriously affected, whereas the resonance angular frequency will be tuned.

\subsection{Experimental measurements}

In order to confirm the theoretical results, the proposed filter has been developed on printed circuit board 

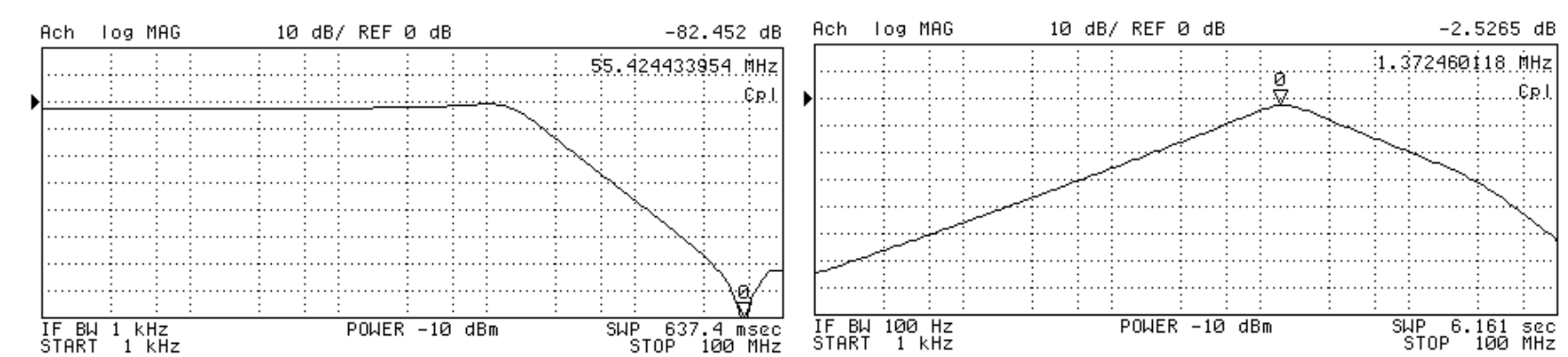

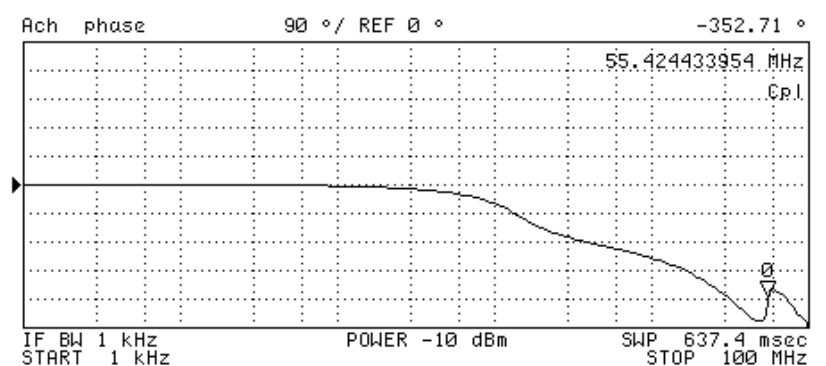

(a)
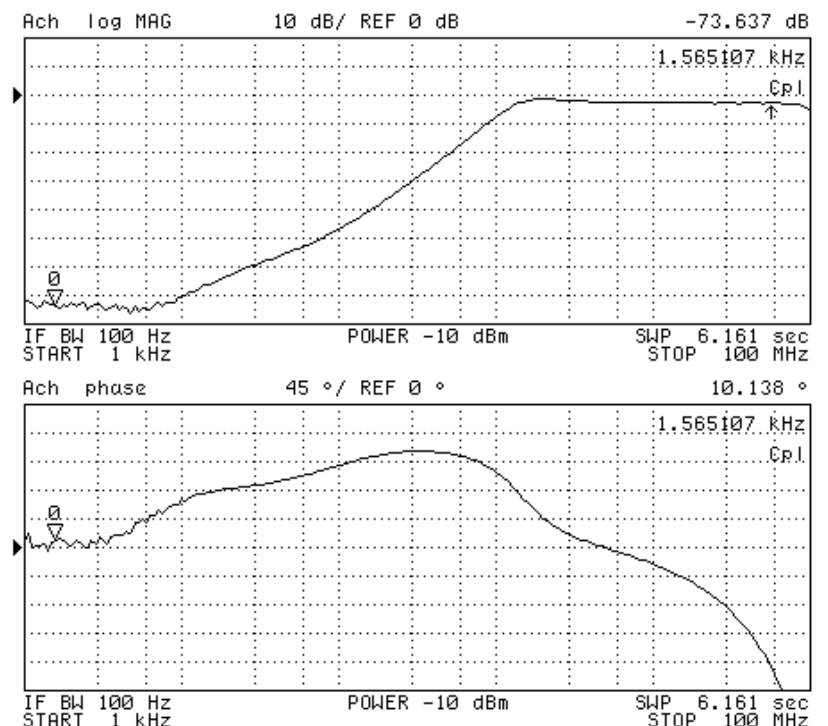

(c)

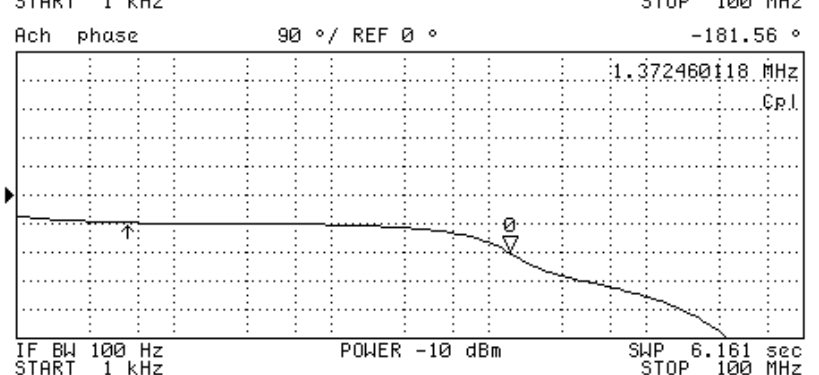

(b)
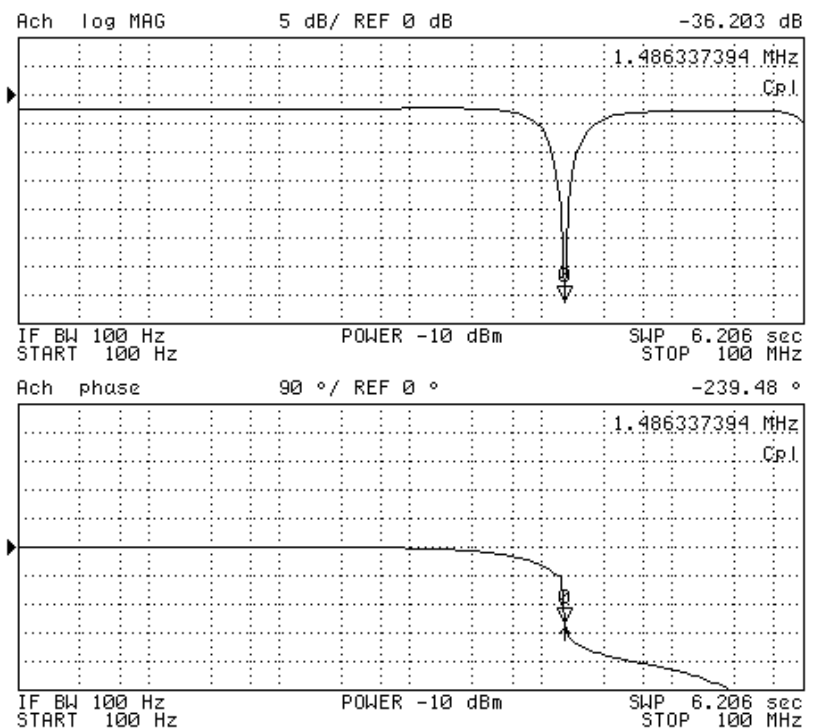

(d)

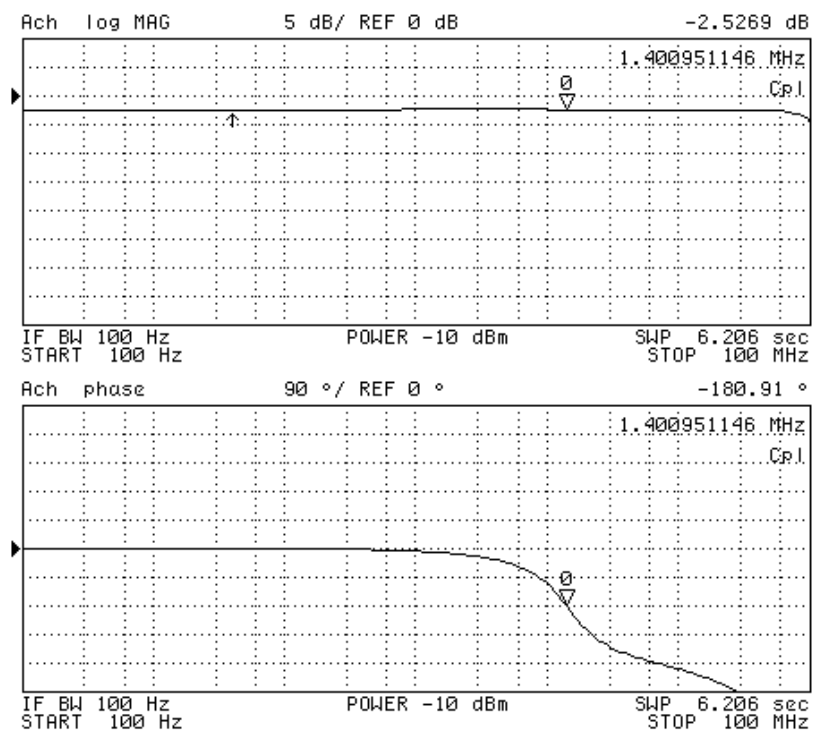

(e)

Fig. 8 Measured magnitude and phase responses of (a) low-pass, (b) band-pass, (c) high-pass, (d) band-reject, and (e) all-pass filters. 
(PCB) and its behavior has been verified by experimental measurements using network-spectrum analyzer Agilent $4395 \mathrm{~A}$. In measurements the VDIBA was implemented based on the structure illustrated in Fig. 7. To realize the input stage of VDIBA the readily available IC OPA660 by BURR BROWN [23] was used, which contains the so-called 'diamond' transistor (DT) and fast voltage buffer (VB). The resistor $R_{A D J}$ was chosen as $220 \Omega$ [23]. In order to increase the linearity of collector current versus input voltage $V_{d}$, the DT is complemented with degeneration resistor $R_{G} \gg 1 / g_{m T}$, added in series to the emitter, where the $g_{m T}$ is the DT transconductance. Then the total transconductance decreases to the approximate value $1 / R_{G}$ [23]. The output stage IVB was realized by IC AD830 produced by Analog Devices [24]. The DC power supply voltages of both ICs were equal to $\pm 5 \mathrm{~V}$. In all measurements the values of the passive components were selected as $C_{1}=C_{2}=100 \mathrm{pF}$ and $R_{G}=R_{\mathrm{MOS}}=1 \mathrm{k} \Omega$ to obtain the designed MISO filter responses with $Q=1$ at pole frequency $f_{0}=\omega_{0} / 2 \pi \cong 1.59 \mathrm{MHz}$. Measured results of $\mathrm{LP}, \mathrm{BP}, \mathrm{HP}, \mathrm{BR}$, and $\mathrm{AP}$ magnitude and phase responses are shown in Fig. 8. From the results it can be observed that due to extra parasitic capacitances of the fabricated $\mathrm{PCB}$ the resonance frequency is $f_{0} \cong 1.48 \mathrm{MHz}$, which, however, is close to the theoretical one.

From the simulation results and experimental measurements it can be seen that the final solution is in good agreement with the theory.

\section{Conclusion}

This letter presents a new VM MISO universal filter to increase the universality of the recently introduced new 'voltage differencing' device so-called voltage differencing inverting buffered amplifier. The proposed circuit is very simple and realizes all five standard filter functions, i.e. LP, BP, HP, BR, and AP in the same circuit topology without need of additional inverting voltage inputs. The proposed filter achieves easy tuning of the resonance angular frequency without seriously affecting the quality factor. In brief, the new filter offers the following advantages: (i) resistorless circuit, (ii) simple structure containing only few transistors, hence, the total transistor area is much smaller that available circuits in the literature, (iii) no component matching is required, (iv) it shows low sensitivity performance, and (v) suitable for low-voltage operation. SPICE simulations and experimental measurements using commercially available ICs confirm the feasibility of the proposed circuit.

\section{Appendix}

This section provides full nomenclature of the aforementioned ABBs in Table 1.

$\begin{array}{ll}\text { OTA } & \begin{array}{l}\text { Operational transconductance amplifier } \\ \text { CFA }\end{array} \\ \text { CCCCTA } & \begin{array}{l}\text { Current feedback amplifier } \\ \text { transconductance amplifier } \\ \text { Current differencing transconductance } \\ \text { amplifier }\end{array} \\ \text { CDTA } & \begin{array}{l}\text { Current-controlled current differencing } \\ \text { buffered amplifier }\end{array} \\ \text { DBTA } & \begin{array}{l}\text { Differential-input buffered and } \\ \text { transconductance amplifier }\end{array} \\ \text { FDCCII } & \begin{array}{l}\text { Fully differential current conveyor } \\ \text { Current follower transconductance }\end{array} \\ \text { CFTA } & \begin{array}{l}\text { amplifier } \\ \text { Second-generation current conveyor }\end{array} \\ \text { CCII } & \text { Differential voltage current conveyor } \\ \text { DVCC } & \text { Voltage differencing buffered amplifier } \\ \text { VDBA } & \text { Differential difference amplifier } \\ \text { DDA } & \text { Voltage differencing inverting buffered } \\ \text { VDIBA } & \text { amplifier }\end{array}$

\section{Acknowledgments}

Ing. Norbert Herencsár, Ph.D. was supported by the project CZ.1.07/2.3.00/30.0039 of Brno University of Technology. Research described in this letter was also in part supported by the project SIX CZ.1.05/2.1.00/ 03.0072 from the operational program Research and Development for Innovation, by the project WICOMT CZ.1.07/2.3.00/20.0007 financed from the operational program Education for competitiveness, BUT Fund No. FEKT-S-11-15, and Czech Science Foundation projects under No. P102/11/P489 and P102/09/1681. Authors also wish to thank the anonymous reviewers for their useful and constructive comments that helped to improve the paper.

\section{References}

1. Sun, Y. (2002). Design of High Frequency Integrated Analogue Filters. London, U.K.: IEE, 184-189.

2. Shah, N. A., \& Rather, M. F. (2006). Voltage-mode OTA based active-C universal filter and its transformation into CFA-based RC-filter. Indian Journal of Pure \& Applied Physics, 44(5), 407-413.

3. Siripruchyanun, M., \& Jaikla, W. (2008). Current controlled current conveyor transconductance amplifier (CCCCTA): a building block for analog signal processing. Electrical Engineering, 90(6), 443-453. 
4. Prasad, D., Bhaskar, D. R., \& Singh, A. K. (2009). Multi-function biquad using single current differencing transconductance amplifier. Analog Integrated Circuits and Signal Processing, 61(3), 309-313.

5. Tangsrirat, W. (2009). Novel minimum-component universal filter and quadrature oscillator with electronic tuning property based on CCCDBAs. Indian Journal of Pure Es Applied Physics, 47(11), 815-822.

6. Herencsar, N., Vrba, K., Koton, J., \& Lattenberg, I. (2009). The conception of differential-input buffered and transconductance amplifier (DBTA) and its application. IEICE Electronics Express, 6(6), 329-334.

7. Kacar, F., Metin, B., Kuntman, H., \& Cicekoglu, O. (2010). A new high-performance CMOS fully differential second-generation current conveyor with application example of biquad filter realisation. International Journal of Electronics, 97(5), 499-510.

8. Tangsrirat, W. (2010). Novel current-mode and voltagemode universal biquad filters using single CFTA. Indian Journal of Engineering \&s Materials Sciences, 17(2), 99104.

9. Chen, H. P. (2010). Single CCII-based voltage-mode universal filter. Analog Integrated Circuits and Signal Processing, 62 (2), 259-262.

10. Kacar, F., \& Yesil, A. (2010). Voltage mode universal filters employing single FDCCII. Analog Integrated Circuits and Signal Processing, 63(1), 137-142.

11. Tangsrirat, W., \& Channumsin, O. (2011). Voltage-mode multifunctional biquadratic filter using single DVCC and minimum number of passive elements. Indian Journal of Pure \& Applied Physics, 49(10), 703-707.

12. Kacar, F., Yesil, A., \& Noori, A. (2012). New CMOS realization of voltage differencing buffered amplifier and its biquad filter applications. Radioengineering, 21(1), 333339

13. Singh, B., Singh, A. K., \& Senani, R. (2013). A new universal biquad filter using differential difference amplifiers and its practical realization. Analog Integrated Circuits and Signal Processing, 75 (2), 293-297.
14. Herencsar, N., Koton, J., Minaei, S., Yuce, E., \& Vrba, K. (2011). Novel resistorless dual-output VM all-pass filter employing VDIBA. In Proceedings of the 7th International Conference on Electrical and Electronics Engineering-ELECO 2011, Bursa, Turkey, pp. 72-74.

15. Herencsar, N., Minaei, S., Koton, J., Yuce, E., \& Vrba, K. (2013). New resistorless and electronically tunable realization of dual-output VM all-pass filter using VDIBA. Analog Integrated Circuits and Signal Processing, $74(1)$, 141-154.

16. Sotner, R., Jerabek, J., \& Herencsar, N. (2013). Voltage differencing buffered/inverted amplifiers and their applications for signal generation. Radioengineering, 22(2), 490-504.

17. Myderrizi, I., Minaei, S., \& Yuce, E. (2011). An electronically fine-tunable multi-inputsingle-output universal filter. IEEE Trans. on Circuits and Systems-II, 58(6), 356360 .

18. Baker, R. J., Li, H. W., \& Boyce, D. E. (1998). CMOS Circuit Design, Layout, and Simulation. New York, USA: IEEE Press, Chapter 7.

19. Fabre, A., Saaid, O., \& Barthelemy, H. (1995). On the frequency limitations of the circuits based on second generation current conveyors. Analog Integrated Circuits and Signal Processing, 7(2), 113-129.

20. Sansen, W. (2006). Analog Design Essentials. Dordrecht, The Netherlands: Springer-Verlag, p. 60.

21. Ogata, K. (2002). Modern Control Engineering. New Jersey: Prentice Hall.

22. TSMC $0.18 \mu \mathrm{m}$ level-7 SCN018 CMOS technology parameters [online]: ftp://ftp.isi.edu/pub/mosis/ vendors/tsmc-018/t44e_lo_epi-params.txt

23. Datasheet OPA660 - Wide Bandwidth Operational Transconductance Amplifier and Buffer. BURR BROWN, SBOS007, 04/1995.

24. Datasheet AD830 - High Speed, Video Difference Amplifier. Analog Devices, Rev. C, 03/2010. 